\title{
WAPL induces cervical intraepithelial neoplasia modulated with estrogen signaling without HPV E6/E7
}

\author{
Katsuyoshi Kumagai ${ }^{1}$ Masakatsu Takanashi $\mathbb{1}^{2} \cdot$ Shin-ichiro Ohno $\mathbb{1}^{2} \cdot$ Yuichirou Harada ${ }^{2} \cdot$ Koji Fujita $^{2}$. \\ Keiki Oikawa ${ }^{2} \cdot$ Katsuko Sudo $^{1} \cdot$ Shun-ichi Ikeda ${ }^{3} \cdot$ Hirotaka Nishi ${ }^{4} \cdot K_{\text {Kosuke Oikawa }}{ }^{5} \cdot$ Masahiko Kuroda $\mathbb{D}^{2}$
}

Received: 21 August 2020 / Revised: 30 March 2021 / Accepted: 12 April 2021 / Published online: 4 May 2021

(c) The Author(s) 2021. This article is published with open access

\begin{abstract}
Since cervical cancer still afflicts women around the world, it is necessary to understand the underlying mechanism of cervical cancer development. Infection with HPV is essential for the development of cervical intraepithelial neoplasia (CIN). In addition, estrogen receptor signaling is implicated in the development of cervical cancer. Previously, we have isolated human wings apart-like (WAPL), which is expected to cause chromosomal instability in the process of HPVinfected precancerous lesions to cervical cancer. However, the role of WAPL in the development of CIN is still unknown. In this study, in order to elucidate the role of WAPL in the early lesion, we established WAPL overexpressing mice (WAPL Tg mice) and HPV E6/E7 knock-in (KI) mice. WAPL Tg mice developed CIN lesion without HPV E6/E7. Interestingly, in WAPL Tg mice estrogen receptor 1 (ESR1) showed reduction as compared with the wild type, but cell growth factors MYC and Cyclin D1 controlled by ESR1 expressed at high levels. These results suggested that WAPL facilitates sensitivity of ESR1 mediated by some kind of molecule, and as a result, affects the expression of MYC and Cyclin D1 in cervical cancer cells. To detect such molecules, we performed microarray analysis of the uterine cervix in WAPL Tg mice, and focused MACROD1, a co-activator of ESR1. MACROD1 expression was increased in WAPL Tg mice compared with the wild type. In addition, knockdown of WAPL induced the downregulation of MACROD1, MYC, and Cyclin D1 but not ESR1 expression. Furthermore, ESR1 sensitivity assay showed lower activity in WAPL or MACROD1 downregulated cells than control cells. These data suggested that WAPL increases ESR1 sensitivity by activating MACROD1, and induces the expression of MYC and Cyclin D1. Therefore, we concluded that WAPL not only induces chromosomal instability in cervical cancer tumorigenesis, but also plays a key role in activating estrogen receptor signaling in early tumorigenesis.
\end{abstract}

Supplementary information The online version contains supplementary material available at https://doi.org/10.1038/s41388021-01787-5.

Masahiko Kuroda

kuroda@tokyo-med.ac.jp

1 Pre-clinical Research Center, Tokyo Medical University, Tokyo, Japan

2 Department of Molecular Pathology, Tokyo Medical University, Tokyo, Japan

3 Department of Obstetrics and Gynecology, Kohseichuo General Hospital, Tokyo, Japan

4 Department of Obstetrics and Gynecology, Tokyo Medical University, Tokyo, Japan

5 Department of Pathology, Wakayama Medical University, Wakayama, Japan

\section{Introduction}

Persistent HPV infection causes cervical squamous epithelial cells to transform into cervical intraepithelial neoplasia (CIN) [1, 2]. In particular, HPV E6/E7 oncoproteins induce immortality and multistep carcinogenesis of cervical squamous epithelial cells $[3,4]$. We have previously reported that HPV E6/E7 increases wings apart-like (WAPL) expression, and WAPL activation in the lesions of CIN and advanced cervical cancer is involved in tumorigenesis and tumor progression $[5,6]$. On the other hand, WAPL is involved in the regulation of the interaction between cohesin and chromatin. WAPL destabilizes cohesin binding to chromatin, but is inhibited in early mitosis by Sororin to maintain stable sister chromatid cohesion. Thus, WAPL is an essential factor for chromatin structure and chromosome segregation [7-12]. Furthermore, we have previously 
shown that high-level expression of WAPL is involved in chromosomal instability [13]. However, it is still unknown whether WAPL plays a direct role in the induction of CIN, which is an early lesion of cervical cancer. Estrogen contributes to the development of cancers such as breast, ovarian, and colorectal cancer by estrogen receptor (ER)dependent and independent mechanisms. In HPVassociated cancer model mouse, estrogen and its receptor estrogen receptor 1 (ESR1) are considered to be essential for the development of cervical cancer [14-16]. ESR1 plays a critical role at the early stage of cervical carcinogenesis [16-19]. However, cervical cancer-derived cell lines and later stage cervical cancer show low-level expression of ESR1 [20, 21]. Furthermore, ESR1 is unnecessary in the process of the progression from high-grade squamous intraepithelial lesions (HSIL)/CIN3 to squamous cell carcinoma (SCC) [20-23]. Therefore, the role of ESR1 in the development of CIN and the significance of ESR1 reduction in CIN3 and SCC are not sufficiently clear. MACROD1 (Mono-ADP-ribosylhydrolase 1) is a macrodomain containing protein that has mono-ADP-ribose hydrolase enzymatic activity, which is also known as leukemia-related protein 16 (LRP16). MACROD1 is a co-activator of ESR1 and upregulates ESR1 target genes including MYC and Cyclin D1 [24]. In addition, knockdown (KD) of MACROD1 in estrogen-resistant ovarian cancer cells markedly reduces estrogen response element-dependent ESR1 reporter gene activity and estrogen-induced MYC expression [25]. However, there have been no reports of an association between MACROD1 and cervical cancer. In this study, we found that WAPL overexpressing mice developed CIN without HPV E6/E7. MACROD1 was enhanced in the uterine cervix of the WAPL overexpressing mice. We also found that KD of WAPL in human cervical cancer cells reduced estrogen sensitivity and expression of ESR1 downstream genes, MYC and Cyclin D1. These data indicated that WAPL associates with MACROD1, influencing the estrogen sensitivity of ESR1 and the expression of MYC and Cyclin D1. Thus, we conclude that WAPL plays a key role in activating estrogen receptor signaling in early tumorigenesis of CIN without involvement of HPV E6/E7.

\section{Results}

\section{Development of CIN in the cervical squamous epithelium by the high-level expression of WAPL without HPV E6/E7}

HPV E6/E7 transgenic (Tg) mice treated with estrogen develop CIN [14]. On the other hand, our previous data have suggested that HPV E6/E7 induce WAPL expression, and overexpression of WAPL in CIN is involved in cervical tumorigenesis and tumor progression [5, 6]. From these results, we considered that WAPL may be implicated in the development of CIN. In this study, to clarify the mechanism of WAPL-mediated cervical carcinogenesis, we examined whether the development of CIN was caused directly by WAPL overexpression instead of the effect of HPV E6/E7. On this account, we generated WAPL transgenic (Tg) mice and HPV E6/E7 knock-in (KI) mice (Supplementary Fig.1).

In rodents, ovarian steroid hormones cause morphological changes in the fallopian tubes, uterus, and upper vagina [26-29]. In order to analyze the association between estrogen and WAPL, WAPL Tg mice need to be suppressed endogenous estrogen secretion and given a certain amount of estrogen. Therefore, we first performed ovariectomy (OVX) to wild type (Wt), HPV E6/E7 KI, and WAPL Tg mice. Then, we examined whether the OVX mice injected with 17ß-Estradiol (E2), the predominant form of estrogen, developed CIN. We failed to find CIN of cervical squamous epithelium in a non-E2-injected group of the mice at 3 and 6 months after OVX (Fig. 1A). In the E2-injected group, however, although abnormalities in the cervical squamous epithelium were not observed in the WAPL Tg mice at 3 months after OVX, the WAPL Tg mice at 6 months after OVX showed dysplastic change of the cervical squamous epithelium (Fig. 1A). Because Ki-67 and P16 are indicators of HSIL/CIN3 [30, 31], screening using a combination of Ki-67 and P16 is expected to be useful for detection of CIN and SCC [32, 33]. Therefore, we performed immunohistochemistry (IHC) analysis using Ki-67 and P16 on the cervical squamous epithelium of the mice. The results showed that the rate of the Ki-67 and P16 positive cells in cervical squamous epithelium of the E2injected WAPL Tg mice at 6 months after OVX was higher than that of the Wt mice (Fig. 1B). Furthermore, the phenotype of the OVX-WAPL Tg mice was severe compared to the E6/E7 KI mice (Fig. 1A). Western blot analysis confirmed that the WAPL expression level in the uterine cervix of the WAPL Tg mice was higher than that of the E6/E7 KI mice (Fig. 1C). These results suggested that the high-level expression of WAPL in the cervical squamous epithelium developed CIN under the effect of estrogen without HPV E6/E7.

\section{Inconsistent expression of ESR1 and its downstream molecules in OVX-WAPL Tg mice}

HPV E6/E7 Tg mice develop CIN by estrogen [14], suggesting that the molecules correlated with HPV E6/E7 are affected by estrogen. On the other hand, we have previously revealed that HPV E6/E7 enhances endogenous WAPL [6]. Moreover, in this study, we demonstrated that WAPL Tg 
Fig. 1 CIN of the cervical squamous epithelium in $\mathrm{OVX}$ treated WAPL Tg mice injected with E2. A HE stain and IHC analysis for Keratin 14 (KRT14) in cervical squamous epithelium of Wt, HPV E6/E7

$\mathrm{KI}$ and WAPL Tg mice at 3 and 6 months after OVX. The mice were divided into two groups, the E2-injected group and nonE2-injected group. The scale bars represent $50 \mu \mathrm{m}$.

B Expression of Ki-67 and P16 in cervical squamous epithelium of E2-injected Wt, HPV E6/E7 $\mathrm{KI}$ and WAPL Tg mice at 6 months after OVX. Left panels show IHC analysis for Ki-67 and P16. Insets show higher magnification images. The scale bars represent $50 \mu \mathrm{m}$. Right panels show the graphs representing the rates of $\mathrm{Ki}-67$ and $\mathrm{P} 16$ positive cells in the cervical squamous epithelium. C Comparison of WAPL protein expression in the uterine cervix between E2-injected HPV E6/E7 $\mathrm{KI}$ and WAPL Tg mice at 6 months after OVX by western blot analysis. The data were obtained from 6 mice for each group, and the representative images are shown. Error bars on the graphs in $\mathbf{B}$ represent SD of the mean. Statistical significance was calculated by Student $t$ test. ${ }^{*} p<0.05$. n.s. no significant difference.
A

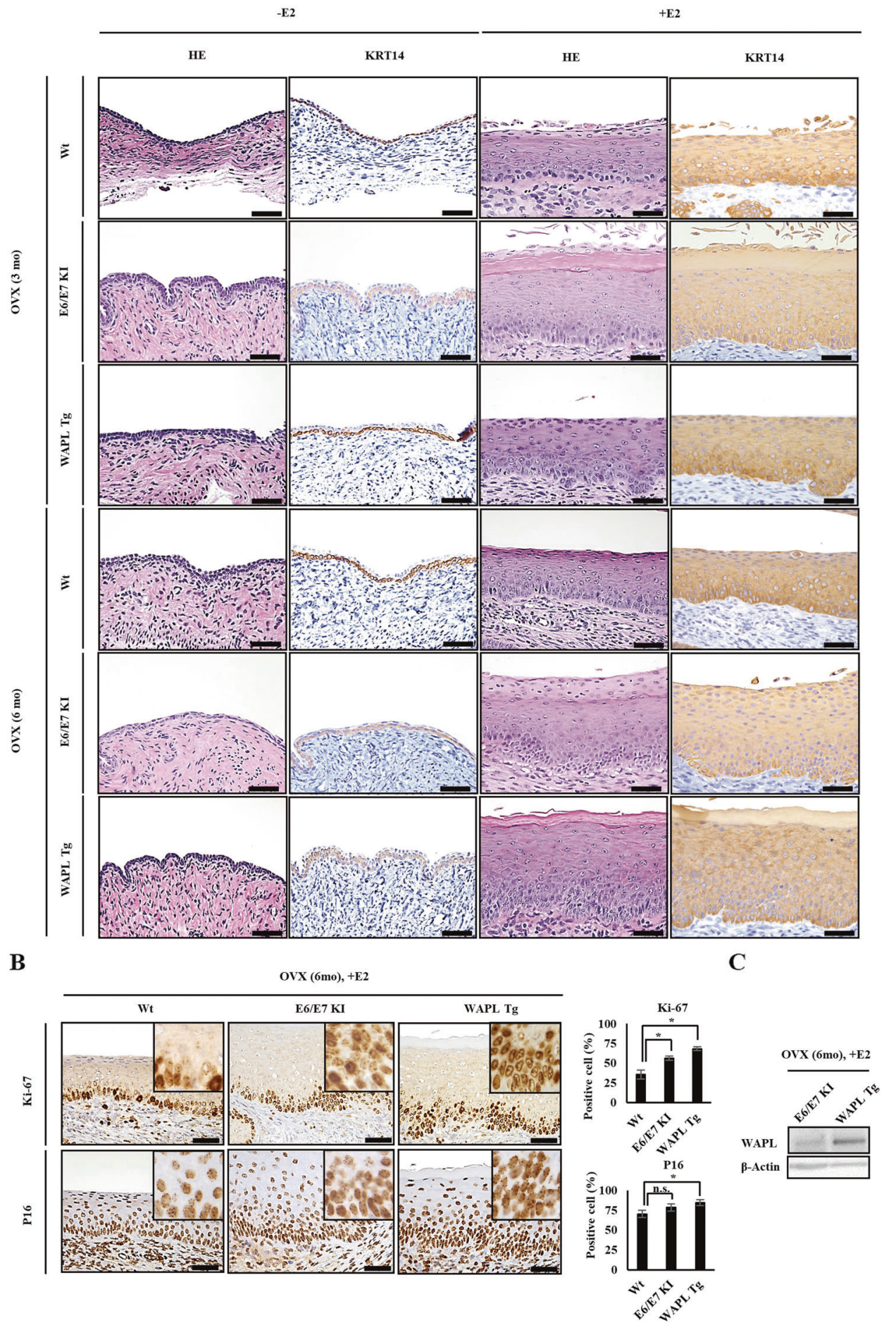

mice developed CIN by estrogen without HPV E6/E7 (Fig. 1). Consequently, WAPL and the estrogen receptor may be correlated. In fact, ESR1 plays a critical role in an early stage of cervical carcinogenesis [16-19]. Therefore, we examined the expression of ESR1 in the cervical squamous epithelium of the E2-injected WAPL Tg mice at 6 months after OVX. Interestingly, the rate of the ESR 1 positive cells in cervical squamous epithelium of the OVX-WAPL Tg mice was significantly lower than that in Wt mice (Fig. 2). Furthermore, we examined the expression of MYC, and Cyclin D1, which is regulated by ESR1 [34]. The rate of the MYC and Cyclin D1 positive cells in cervical squamous 

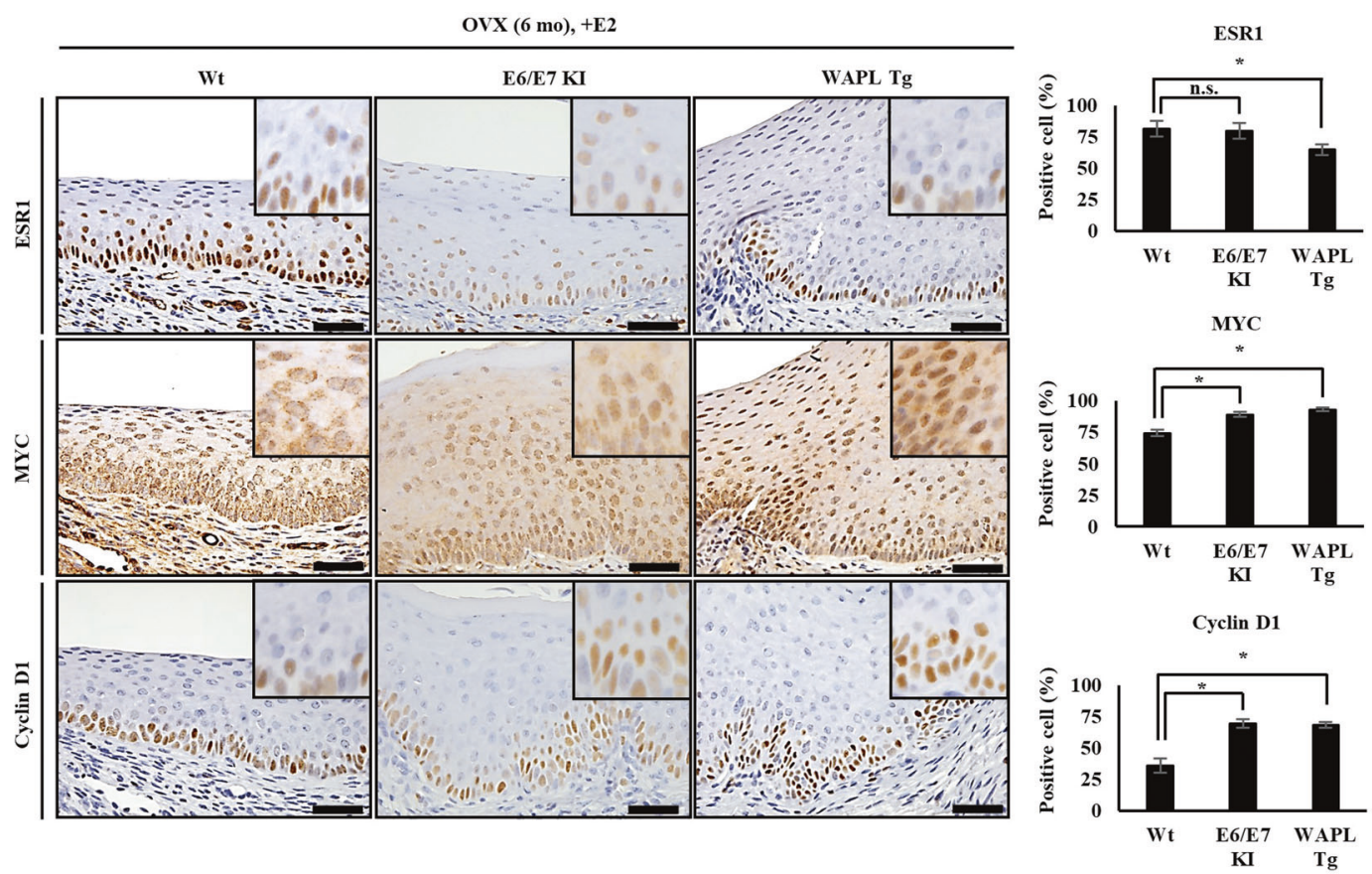

Fig. 2 Comparison of the expression of ESR1, MYC, and Cyclin D1 in the cervical squamous epithelium among E2-injected Wt, HPV E6/E7 KI and WAPL Tg mice at 6 months after OVX. Left panels show IHC analysis of ESR1, MYC, and Cyclin D1. Insets show higher magnification images. The scale bars represent $50 \mu \mathrm{m}$. Right panels show the graphs representing the rates of ESR1, MYC, and

epithelium of the OVX-WAPL Tg mice was significantly higher than that in Wt mice (Fig. 2).

\section{Detection of the molecule affecting ESR1 by the high-level expression of WAPL}

In general, ESR1 expression causes induction of MYC and Cyclin D1. In the above results, however, the expression of MYC and Cyclin D1 in WAPL Tg mice was higher than that of Wt mice, although the ESR1 expression in WAPL Tg mice was significant lower (Fig. 2). Thus, we considered that some kind of molecule enhanced the sensitivity of ESR1 to estrogen by the high-level expression of WAPL. Therefore, to identify such molecules, we performed DNA microarray analysis of gene expression in the uterine cervix of WAPL Tg mice and Wt mice (Fig. 3A). As a result, 607 molecules were highly expressed in WAPL Tg mice compared to Wt mice (Supplemental Table S1). Among them, four factors, IGF1R, IRS2, FOXO4, and MACROD1, are associated with ESR1. Moreover, we focused on MACROD1, which interacts with ESR1 and regulates the expression of MYC and Cyclin D1 [24, 35]. Then, to confirm MACROD1 expression in the uterine cervix of WAPL Tg mice, we performed RT-PCR and western blot analysis. As a result, MACROD1 was significantly higher in WAPL Tg mice than Wt mice (Fig. 3B, C).
Cyclin D1 positive cells in the cervical squamous epithelium. The data were obtained from 6 mice for each group, and the representative images are shown. Error bars on the graphs represent S.D. of the mean. Statistical significance was calculated by Student $t$ test. $* p<0.05$. n.s. no significant difference.

\section{MACROD1 expression is correlated with WAPL in human cervical cancer cells}

MACROD1 is a co-activator of ESR1, and responds to estrogen [24]. However, there are no reports indicating that MACROD1 is associated with the development of cervical cancer. Therefore, to investigate the correlation between MACROD1 and WAPL, we examined the effects of WAPL KD on MACROD1 in a human cervical SCC cell line SiHa and a human cervical adenocarcinoma cell line HeLa. As a result, ESR1 expression showed no significant difference between the WAPL KD cells and non-target KD cervical cancer cells (control cells), but the expression of MACROD1 in the WAPL KD cells was significantly decreased as compared to the control cells (Fig. 4A, B). In addition, we observed a decreased expression of MYC and Cyclin D1 in WAPL KD cells (Fig. 4A, B). These results suggested a correlation between WAPL and MACROD1. Therefore, to further study the correlation WAPL and MACROD1, we overexpressed MACROD1 in WAPL KD-SiHa and -HeLa cells and examined the recovery of expression of MYC or Cyclin D1 with and without E2. The expression of MYC and Cyclin D1 was not induced by MACROD1 overexpression in WAPL KD cells without E2, but was induced with E2 (Fig. 4C). Furthermore, we examined the effects of MACROD1 KD on $\mathrm{SiHa}$ and HeLa cells. Although WAPL and ESR1 expression 
Fig. 3 Identification of an

ESR1-related factor

MACROD1 in the uterine cervix of 6-month-old WAPL

Tg mice. A Schematic

procedure of DNA microarray

analysis to obtain the genes that

are differentially expressed

twofold or more higher in the

uterine cervix of WAPL Tg mice

relative to $\mathrm{Wt}$ mice.

B Comparison of WAPL and

MACROD1 mRNA expression

in the uterine cervix among 6month-old Wt, HPV E6/E7 KI, and WAPL Tg mice by quantitative real-time PCR.

C Comparison of WAPL and

MACROD1 protein expression

in the uterine cervix among 6-

month-old Wt, HPV E6/E7 KI,

and WAPL Tg mice by western

blot analysis. B, C Data were

obtained from six mice for each

group. Error bars on the graphs

represent $\mathrm{SD}$ of the mean.

Statistical significance was

calculated by Student $t$ test.

${ }^{*} p<0.05$. n.s. no significant

difference.
A

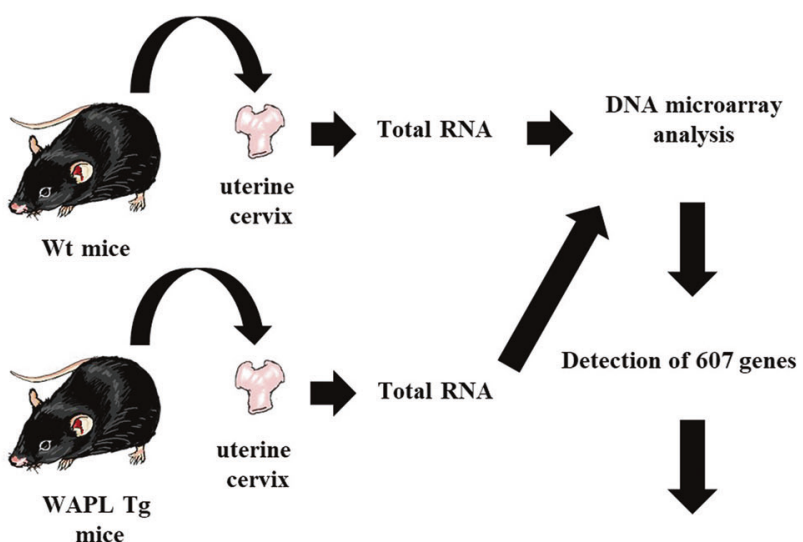

Identification of MACROD1 gene

B

WAPL

MACROD1
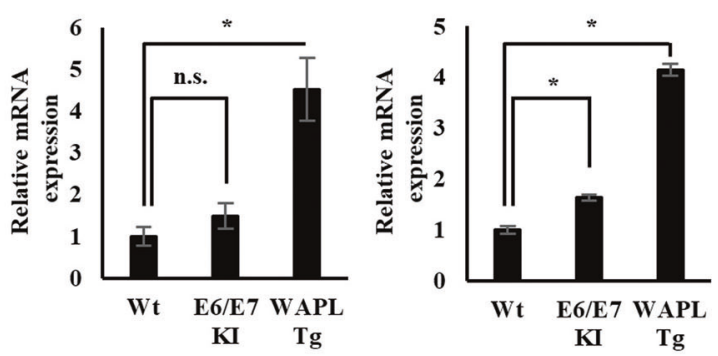

C
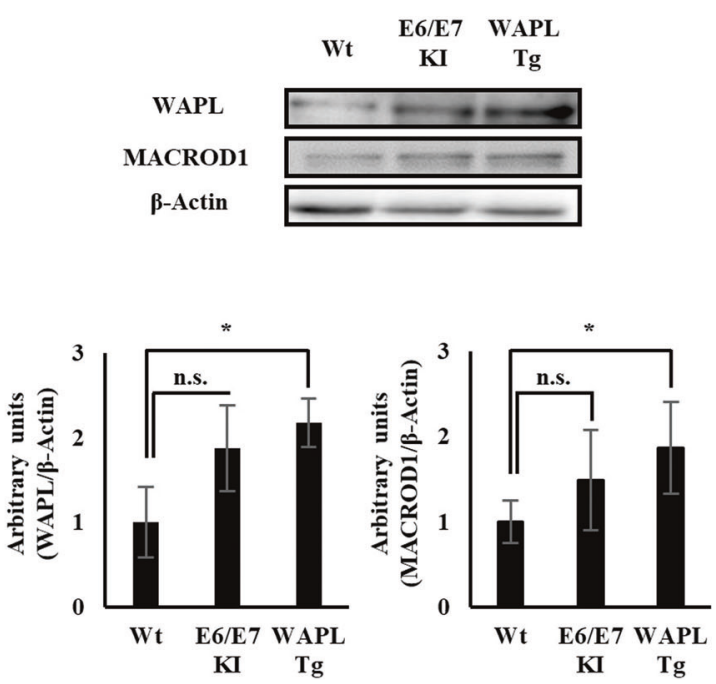

WAPL increases ESR1 sensitivity to estrogen by activating MACROD1 in human cervical cancer cells

As shown in Fig. 2, we found high-level expression of MYC and Cyclin D1 in the cervical squamous epithelium of WAPL Tg mice even though ESR1 exhibited low-level expression. Moreover, MACROD1 showed high-level showed no significant difference between the MACROD1 KD cells and control cells, the expression of MYC and Cyclin D1 in the MACROD1 KD cells was significantly decreased as compared to the control cells (Fig. 4D). These results suggested that MACROD1 is regulated by WAPL, and affects the activity of ESR1 downstream molecules such as MYC and Cyclin D1 in human cervical cancer cells. 
A

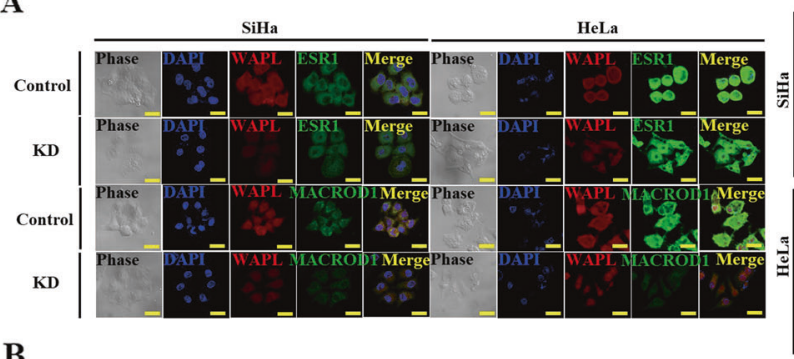

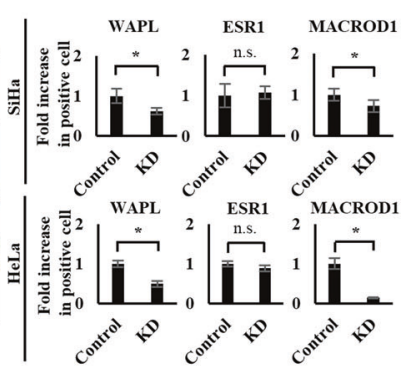

ESR1

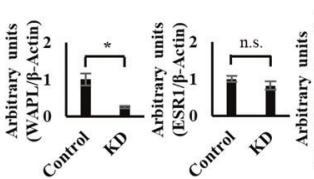
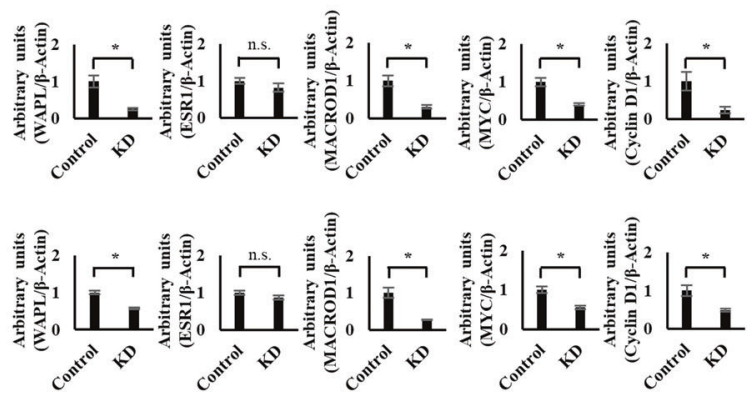

C

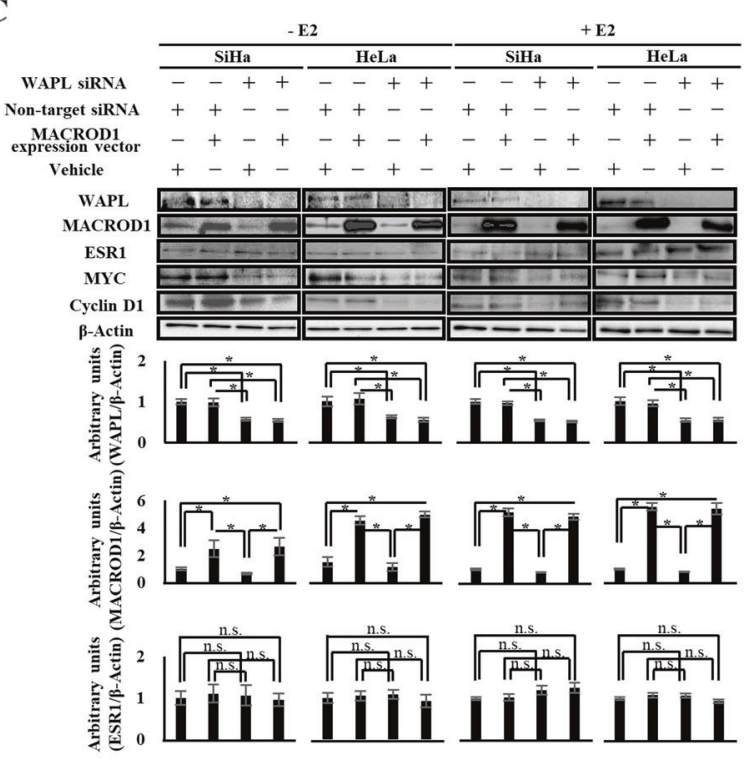

D

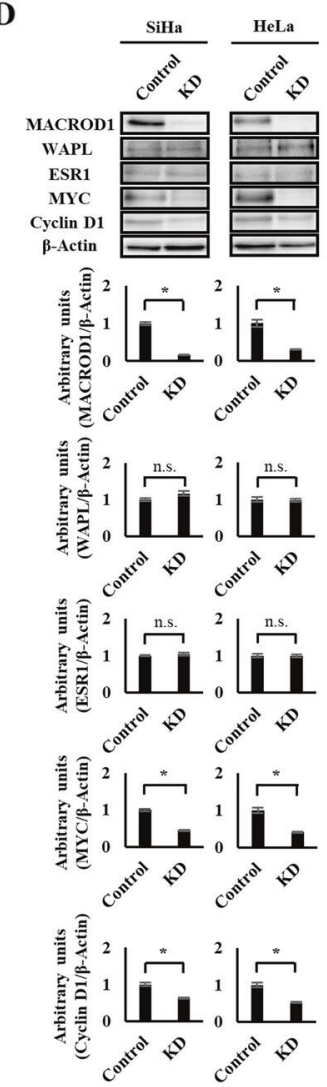

expression in WAPL Tg mice (Fig. 3B). Furthermore, the expression of MYC, Cyclin D1, and MACROD1 was decreased in cervical cancer cells by WAPL KD although there was no significant difference of ESR1 expression level between WAPL KD and control cells (Fig. 4A-C). From these results, we speculated that WAPL may increase
ESR1 sensitivity to estrogen by activating MACROD1. Then, we performed luciferase-based E2-ESR1 reporter assay using WAPL KD and MACROD1 KD human cervical cancer cells. The luciferase activity in E2-treated WAPL KD cells was significantly lower than that in control cells (Fig. 5). Surprisingly, the luciferase activity in E2- 
Fig. 4 Correlation between WAPL and MACROD1 in human cervical cancer cells, SiHa and HeLa. A Immunofluorescence analysis of WAPL, ESR1, and MACROD1 in WAPL knockdown (KD) and non-targeted knockdown (control) cells. Left panel shows representative images of Immunofluorescence analysis from five samples three independent experiments. The scale bars represent $20 \mu \mathrm{m}$. Right panels show the graphs representing the ratios of WAPL, ESR1, and MACROD1 positive cells between WAPL KD and control cells. B Western blot analysis of WAPL, ESR1, MACROD1, MYC, and Cyclin D1 proteins in WAPL KD and control cells. Left panels show representative images of western blot analysis from six samples in each group and three independent experiments. Right panels show the graphs representing the intensities of signals of western blot analysis. C Effect of MACROD1 overexpression on MYC and cyclin D1 expression in WAPL KD cells with and without E2. WAPL siRNA, non-target siRNA, MACROD1 expression vector, and/or vehicle were transfected into $\mathrm{SiHa}$ and HeLa cells as indicated above the blot panels. One day after MACROD1 transfection, the cells were treated with or without $100 \mathrm{nM}$ of E2. Then, 2 days after transfection, cells were harvested and subjected to western blot analysis. Upper panels show representative images of western blot analysis from six samples in each group and three independent experiments. Lower panels show graphs representing intensities of signals of western blot analysis. D Effect of MACROD1 KD on the expression of WAPL, ESR1, MYC, and Cyclin D1 proteins in cervical cancer cells. Upper panels show representative images of western blot analysis from six samples in each group and three independent experiments. Lower panels show graphs representing intensities of signals of western blot analysis. Error bars on the graphs represent SD of the mean. Statistical significance was calculated by Student $t$ test. ${ }^{*} p<0.05$, n.s. no significant difference.

treated MACROD1 KD cells was also significantly lower than that in control cells (Fig. 5). These results suggested that WAPL increases ESR1 sensitivity to estrogen by activating MACROD1 in human cervical cancer cells.

\section{MACROD1 expression shows a similar expression pattern to WAPL in human cervical lesions}

In the present study, we showed that WAPL induced MACROD1 expression in the experiments using animal models and human cultured cells. Next, we examined the expression patterns of MACROD1 and WAPL in human cervical lesions by immunostaining. WAPL and MACROD1 showed similar expression patterns in CIN1, CIN2-3, and SCC (Fig. 6A). Furthermore, the Pearson correlation coefficient indicated that MACROD1 expression was positively correlated with WAPL expression in human cervical lesions (Fig. 6B).

\section{Discussion}

Persistent infection with HPV causes CINs in uterine cervix, leading to advanced cervical cancer. In this case, HPV E6/E7 oncoproteins bind to the tumor suppressors p53 and $\mathrm{pRB}$, respectively, resulting in the degradation of p53 and
SiHa

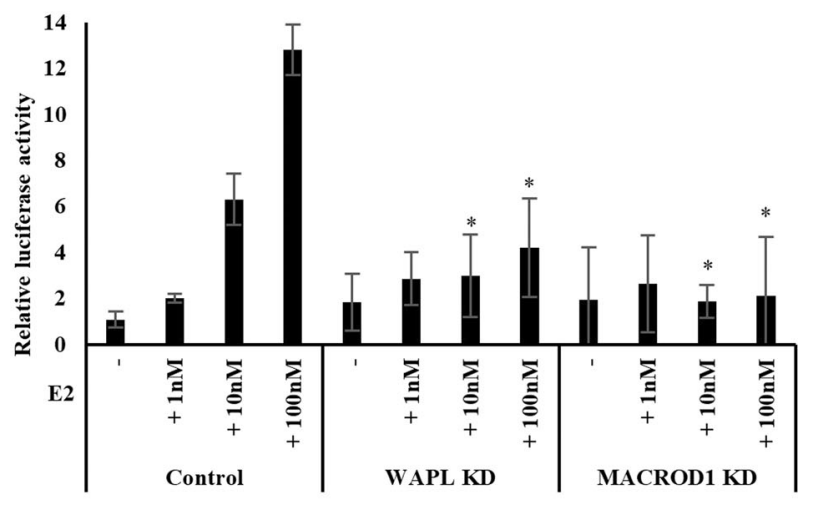

HeLa

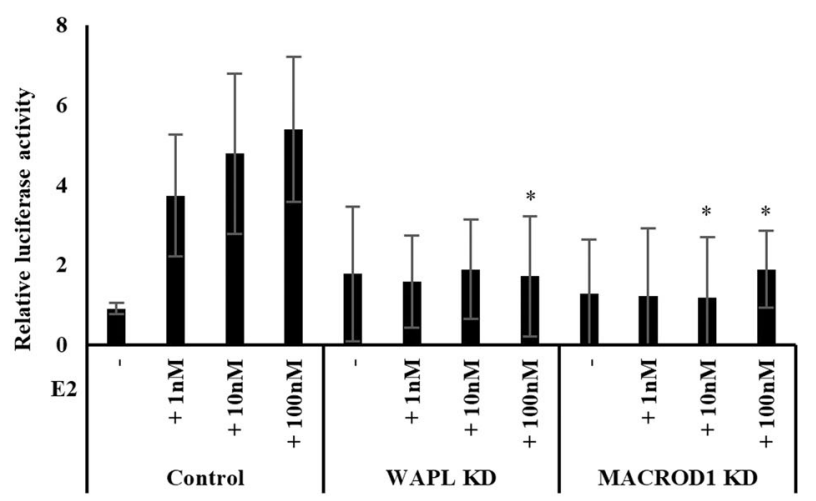

Fig. 5 Analysis of ESR1 sensitivity to estrogen in WAPL and MACROD1 KD-SiHa and HeLa cells by luciferase reporter assay. Control is non-targeted knockdown in the cells. Error bars on the graphs represent SD of the mean from six samples in each group and three independent experiments. Statistical significance was calculated by Student $t$-test. ${ }^{*} p<0.05$.

pRB via ubiquitin. Therefore, the carcinogenesis progresses in the same state as the accumulation of mutations in the p53 and pRB genes [36, 37]. We have previously reported that WAPL is induced by HPV E6/E7, is activated in patients with CIN and advanced cervical cancer $[5,6]$ and causes chromatin instability [13]. However, the mechanism by which activated WAPL progresses CIN and cervical cancer is not fully understood at in vivo level. In this study, we generated WAPL Tg mice overexpressing WAPL in the uterine cervix. We found that high-level expression of WAPL induced CIN in cervical squamous epithelium. On the other hand, accumulation of DNA damage in HPV E6/ E7-expressing cells leads to the cell cycle progression and the formation of anaphase bridges between chromosomes during the anaphase of cell division, leading to chromosomal structural abnormalities [38, 39]. Thus, continuous expression of HPV E6/E7 may lead to the accumulation of chromosomal aberrations and high-level expression of WAPL, which may induce cell transformation in uterine 


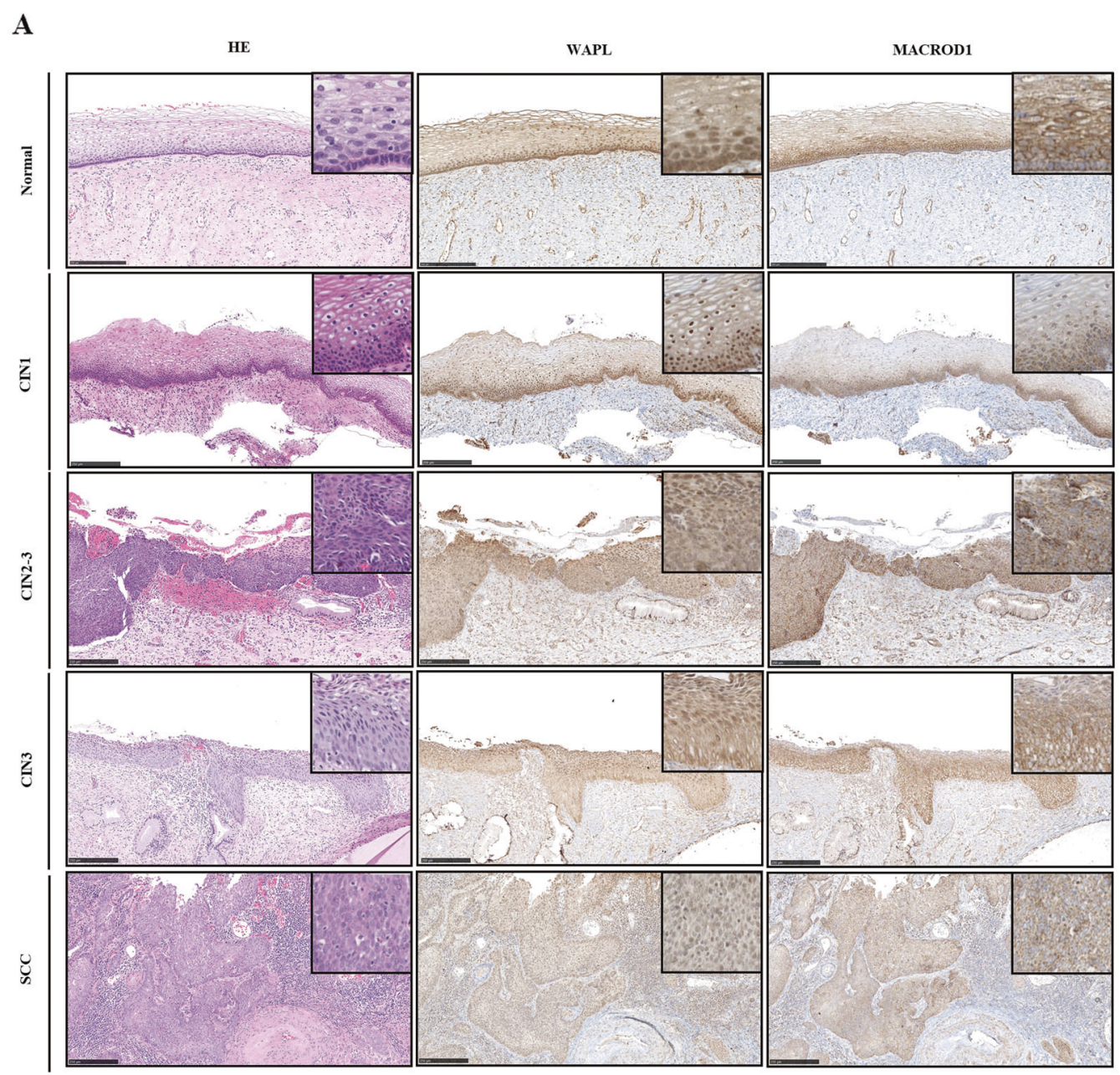

B

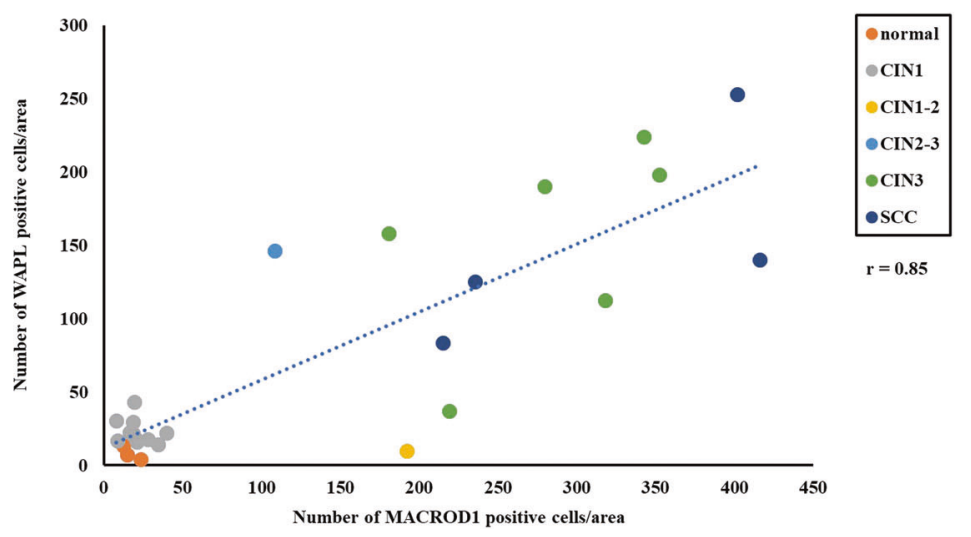

Fig. 6 WAPL and MACROD1 expression patterns in human cervical squamous epithelial lesions. A HE stain and IHC analysis of WAPL and MACROD1 in Normal, CIN1, CIN2-3, CIN3, and SCC. The scale bars represent $250 \mu \mathrm{m}$. B Liner correlation between WAPL and MACROD1 positive cells in IHC analysis. Straight lines are fitted

cervix. K14-E6/E7 Tg mice, which highly express E6/E7 under the control of the human keratin 14 promoter, develop invasive cancer [40, 41]. However, in the present to the data by linear regression with Pearson's correlation coefficients $r=0.85, p<0.01$. The data were obtained from three patients with normal, 12 patients with CIN1, 1 patient with CIN1-2, 1 patient with CIN2-3, 6 patients with CIN3, and 4 patients with SCC for each group.

study, E6/E7 KI mice developed CIN but did not lead to cervical cancer. Thus, we consider that the difference of the expression level of E6/E7 in cervical squamous epithelial 
Fig. 7 The proposed of mechanism for CIN. In HPVinfected cervical epithelial cells, WAPL plays key roles in the regulation of estrogen sensitivity by activating MACROD1 in early stages of carcinogenesis.

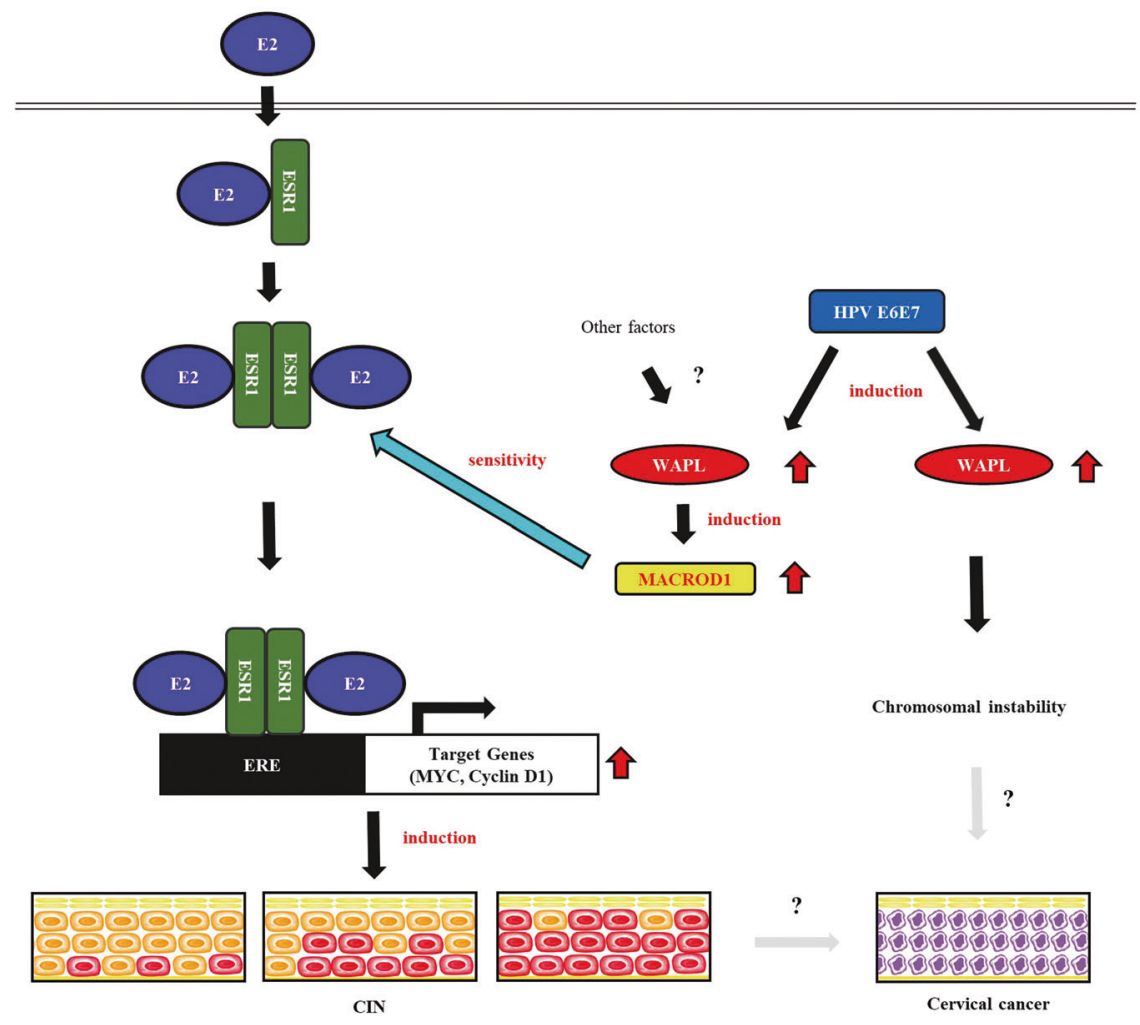

cells between K14-E6/E7 Tg mice and E6/E7 KI mice may have caused a difference in the expression level of WAPL induced by E6/E7. In fact, although our previous study has demonstrated that exogenous expression of E6/E7 induce WAPL [6], WAPL expression level in E6/E7 KI mice was not significantly higher than that in Wt mice. We also found that high-level expression of WAPL in the cervix caused CIN. The symptoms were more severe than those of E6/E7 KI mice. In addition, WAPL expression in WAPL Tg mice was higher than that in E6/E7 KI mice, suggesting that the transformation of cervical squamous epithelial cells is WAPL-dependent. Furthermore, we have revealed that WAPL expression pattern is significantly correlated with the severity of human cervical lesions [5]. The expression pattern was also confirmed in the present study. Thus, WAPL may play a key role in cervical oncogenesis.

On the other hand, the transcription factor SALL4 is highly activated in various malignant tumors including cervical, liver, lung, gastric, breast, and endometrial cancers [42-47]. SALL4 activates MYC and Cyclin D1 by upregulating the $\mathrm{Wnt} / \beta$-catenin pathway in cervical cancer cells [42]. In addition, other transcription factors such as NANOG, OCT4, LGR5, and EZH2 promote tumor formation in cervical cancer [48-51]. Thus, these transcription factors may be elaborately associated with WAPL and ESR1. In fact, increased expression of LGR5 was observed in WAPL Tg mice compared to Wt mice by DNA microarray analysis. However, we failed to detect SALL4,
NANOG, and OCT4 in human cervical cancer tissues by immunostaining analysis (data not shown). Thus, further experiments are required to elucidate the mechanism. Genes and transcription factors under the control of estrogen receptors are involved in the formation of cervical squamous epithelium [52, 53]. On the other hand, estrogen and its receptors are risk factors that contribute to the development and malignancy of CIN and cervical cancer $[14,15,17,54]$. In this study, we analyzed the association between estrogen and its receptor in the development of CIN. We found that WAPL increased the estrogen sensitivity of ESR1 by activating MACROD1. Moreover, MACROD1 showed a similar expression pattern to WAPL in various human cervical specimens including normal epithelium, CIN and SCC. On the other hand, overexpression of MACROD1 activates RAC1 and ERK1/2, which are heavily involved in cell proliferation and oncogenic mechanisms [55]. Thus, we concluded that WAPL not only induces chromosomal instability in cervical carcinogenesis, but also plays a key role in the regulation of estrogen sensitivity by activating MACROD1 in early stages of carcinogenesis (Fig. 7). Further analysis of WAPL and MACROD1 may provide a new preventive measure against development of CIN. In conclusion, this study demonstrates that high-level expression of WAPL extends CIN in the cervical squamous epithelium of WAPL Tg mice. Furthermore, WAPL increases ESR1 sensitivity to estrogen by activating MACROD1, and influences the 
expression of the ESR1 downstream factors MYC and cyclin D1. Thus, we have uncovered a part of the roles of WAPL in the development of CIN and cervical cancer.

\section{Materials and methods}

\section{MACROD1 expression vector and transfection}

The full length of MACROD1 cDNA derived from human non-small cell lung carcinoma cell line H1299 was inserted into the pcDNA 3.1 vector (Thermo Fisher Scientific). The resultant MACROD1 expression vector was transfected into $\mathrm{SiHa}$ or HeLa cells using Lipofectamine 3000 reagent (Thermo Fisher Scientific) according to the manufacturer's recommendation. Subsequently, $100 \mathrm{nM}$ of E2 was added to the cells 1 day after transfection. The cells were collected at $24 \mathrm{~h}$ after the addition of E2. The detailed information of the primers to amplify or confirm MACROD1 sequence is described in Supplemental Table S2.

\section{Animals}

All animal care and procedures that were performed in this study conformed to the guidelines for animal experiments of Tokyo Medical University, and they were approved by the Animal Research Committee of Tokyo Medical University (R2-0066). The mice with developmental abnormalities were excluded from experiments. In addition, normal individuals were randomly selected.

\section{WAPL Tg mice and HPV E6/E7 KI mice}

Further details are provided in the Supplementary materials and methods.

\section{Immunohistochemistry (IHC) analysis}

All human cervical specimens were obtained from patients treated at Tokyo Medical University Hospital (Tokyo, Japan) under an approved Institutional Review Board protocol (T2020-0324). The human and murine uterine cervix specimens were fixed with formalin and embedded in paraffin. Sections were stained with Hematoxylin and Eosin or incubated with a primary antibody, and a second antibody. They were visualized using the EnVision DAB kit (Dako A/S, Denmark). Stained images were taken and analyzed by NanoZoomer Digital Pathology software (Hamamatsu Photonics, Japan). The specific information of antibodies is described in Supplemental Table S3. Six mice in each group for scoring of IHC staining, IHC staining cells were counted from five locations randomly selected from the section of the uterine cervix of WAPL transgenic mice and human uterine cervix by ImageJ software. Either nucleus or cytoplasmic signal above background was scored as positive.

\section{Immunofluorescence microscopy}

Three days after transfection of WAPL siRNA, SiHa and HeLa cells were fixed with $1 \%$ glutaraldehyde and methanol. After blocking with $1 \%$ BSA, the samples were incubated sequentially with primary and secondary antibodies. Then, the samples were analyzed by LSM 710 laser scan microscope (Carl Zeiss, Germany). Fluorescence intensities from images of 5-8 randomly selected microscopic fields of samples were semi-quantitatively analyzed by ZEN lite software (Carl Zeiss). The specific information of antibodies is described in Supplemental Table S3.

\section{Luciferase reporter assays}

$25 \mathrm{nM}$ of WAPL siRNA, MACROD1 siRNA, or negative control siRNA was transfected into $\mathrm{SiHa}$ and HeLa cells. The next day, pGL4 luciferase reporter vector (Promega, USA) containing estrogen response element-dependent ESR1 was transfected into the cells using the Lipofectamine 3000 reagent (Thermo Fisher). Then, 1, 10, or $100 \mathrm{nM}$ of E2 were added to the cells 2 days after siRNA transfection. The cell extracts were prepared $24 \mathrm{~h}$ after the addition of E2, and the luciferase activity was measured using the Dual-Luciferase Reporter Assay System (Promega) according to the manufacturer's recommendation. All experiments were performed in triplicate.

\section{Data availability}

Access to the data from our study is available upon reasonable request

Acknowledgements We thank Dr. Shouichirou Mineo of Tokyo Medical University and Dr. Shinichi Miyagawa of Tokyo University of Science for their technical assistance and advice regarding the experiments. We appreciate Dr. Tohru Kiyono of the National Cancer Center Research Institute for his donating of pDONR201-16E6E7 vector. This work was partly supported by the Grants-in-Aid for Scientific Research of Education, Culture, Sports, Science and Technology, Japan (MEXT Grant), Grant number: (20K09654 to KK) and the Tokyo Medical University Foundation Cancer Research Fund.

Author contributions KK and MK proposed the concept, contributed to the study design, performed the experiments, statistical analyzed and wrote the manuscript. KF contributed to the provided technical support for collection and interpretation of pathological and immunohistochemical data. $\mathrm{SO}, \mathrm{YH}, \mathrm{KO}$, and $\mathrm{KS}$ contributed to the collection and interpretation of molecular data and the critical discussion of the study. SI and HN advised on clinical research. MT and KO assisted with experimental design, data analysis, and writing the manuscript. 


\section{Compliance with ethical standards}

Conflict of interest The authors declare no competing interests.

Publisher's note Springer Nature remains neutral with regard to jurisdictional claims in published maps and institutional affiliations.

Open Access This article is licensed under a Creative Commons Attribution 4.0 International License, which permits use, sharing, adaptation, distribution and reproduction in any medium or format, as long as you give appropriate credit to the original author(s) and the source, provide a link to the Creative Commons license, and indicate if changes were made. The images or other third party material in this article are included in the article's Creative Commons license, unless indicated otherwise in a credit line to the material. If material is not included in the article's Creative Commons license and your intended use is not permitted by statutory regulation or exceeds the permitted use, you will need to obtain permission directly from the copyright holder. To view a copy of this license, visit http://creativecommons. org/licenses/by/4.0/

\section{References}

1. Snijders PJ, Steenbergen RD, Heideman DA, Meijer CJ. HPVmediated cervical carcinogenesis: concepts and clinical implications. J Pathol. 2006;208:152-64.

2. Steenbergen RD, Snijders PJ, Heideman DA, Meijer CJ. Clinical implications of (epi)genetic changes in HPV-induced cervical precancerous lesions. Nat Rev Cancer. 2014;14:395-405.

3. Harald zur H. Papillomaviruses and cancer: from basic studies to clinical application. Nat Rev Cancer. 2002;2:342-50.

4. Narisawa-Saito M, Kiyono T. Basic mechanisms of high-risk human papillomavirus-induced carcinogenesis: roles of E6 and E7 proteins. Cancer Sci. 2007;98:1505-11.

5. Oikawa K, Ohbayashi T, Kiyono T, Nishi H, Isaka K, Umezawa $\mathrm{A}$, et al. Expression of a novel human gene, human wings apartlike (hWAPL), is associated with cervical carcinogenesis and tumor progression. Cancer Res. 2004;64:3545-9.

6. Kuroda M, Kiyono T, Oikawa K, Yoshida K, Mukai K. The human papillomavirus E6 and E7 inducible oncogene, hWAPL, exhibits potential as a therapeutic target. $\mathrm{Br} \mathrm{J}$ Cancer. 2005;92:290-3.

7. Gandhi R, Gillespie PJ, Hirano T. Human Wapl is a cohesinbinding protein that promotes sister-chromatid resolution in mitotic prophase. Curr Biol. 2006;16:2406-17.

8. Kueng S, Hegemann B, Peters BH, Lipp JJ, Schleiffer A, Mechtler $\mathrm{K}$, et al. Wapl controls the dynamic association of cohesin with chromatin. Cell. 2006;127:955-67.

9. Nishiyama T, Ladurner R, Schmitz J, Kreidl E, Schleiffer A, Bhaskara V, et al. Sororin mediates sister chromatid cohesion by antagonizing Wapl. Cell. 2010;143:737-49.

10. Zhang N, Pati D. Sororin is a master regulator of sister chromatid cohesion and separation. Cell Cycle. 2012;11:2073-83.

11. Hara K, Zheng G, Qu Q, Liu H, Ouyang Z, Chen Z, et al. Structure of cohesin subcomplex pinpoints direct shugoshin-Wapl antagonism in centromeric cohesion. Nat Struct Mol Biol. 2014;21:864-70.

12. Ladurner R, Kreidl E, Ivanov MP, Ekker H, Idarraga-Amado MH, Busslinger GA, et al. Sororin actively maintains sister chromatid cohesion. EMBO J. 2016;35:635-53.

13. Ohbayashi T, Oikawa K, Yamada K, Nishida-Umehara C, Matsuda Y, Satoh H, et al. Unscheduled overexpression of human WAPL promotes chromosomal instability. Biochem Biophys Res Commun. 2007;356:699-704.
14. Arbeit JM, Howley PM, Hanahan D. Chronic estrogen-induced cervical and vaginal squamous carcinogenesis in human papillomavirus type 16 transgenic mice. Proc Natl Acad Sci USA. 1996;93:2930-5.

15. Chung SH, Franceschi S, Lambert PF. Estrogen and ERalpha: culprits in cervical cancer? Trends Endocrinol Metab. 2010;21:504-11.

16. Chung SH, Lambert PF. Prevention and treatment of cervical cancer in mice using estrogen receptor antagonists. Proc Natl Acad Sci USA. 2009;106:19467-72.

17. Chung SH, Wiedmeyer K, Shai A, Korach KS, Lambert PF. Requirement for estrogen receptor alpha in a mouse model for human papillomavirus-associated cervical cancer. Cancer Res. 2008;68:9928-34.

18. Son J, Park JW, Lambert PF, Chung SH. Requirement of estrogen receptor alpha DNA-binding domain for HPV oncogene-induced cervical carcinogenesis in mice. Carcinogenesis. 2014;35:489-96.

19. Son J, Park Y, Chung SH. Epithelial oestrogen receptor $\alpha$ is dispensable for the development of oestrogen-induced cervical neoplastic diseases. J Pathol. 2018;245:147-52.

20. Zhai Y, Bommer GT, Feng Y, Wiese AB, Fearon ER, Cho KR. Loss of estrogen receptor 1 enhances cervical cancer invasion. Am J Pathol. 2010;177:884-95.

21. Kwasniewska A, Postawski K, Gozdzicka-Jozefiak A, Kwasniewski W, Grywalska E, Zdunek M, et al. Estrogen and progesterone receptor expression in HPV-positive and HPV-negative cervical carcinomas. Oncol Rep. 2011;26:153-60.

22. den Boon JA, Pyeon D, Wang SS, Horswill M, Schiffman M, Sherman M, et al. Molecular transitions from papillomavirus infection to cervical precancer and cancer: role of stromal estrogen receptor signaling. Proc Natl Acad Sci USA. 2015;112:E3255-64.

23. Hong MK, Wang JH, Su CC, Li MH, Hsu YH, Chu TY. Expression of estrogen and progesterone receptor in tumor stroma predicts favorable prognosis of cervical squamous cell carcinoma. Int J Gynecol Cancer. 2017;27:1247-55.

24. Han WD, Zhao YL, Meng YG, Zang L, Wu ZQ, Li Q, et al. Estrogenically regulated LRP16 interacts with estrogen receptor alpha and enhances the receptor's transcriptional activity. Endocr Relat Cancer. 2007;14:741-53.

25. Tian L, Wu Z, Zhao Y, Meng Y, Si Y, Fu X, et al. Differential induction of LRP16 by liganded and unliganded estrogen receptor alpha in SKOV3 ovarian carcinoma cells. J Endocrinol. 2009;202:167-77.

26. Merkwitz C, Blaschuk O, Eplinius F, Winkler J, Prömel S, Schulz $\mathrm{A}$, et al. A simple method for inducing estrous cycle stage-specific morphological changes in the vaginal epithelium of immature female mice. Lab Anim. 2016;50:344-53.

27. Goldman JM, Murr AS, Cooper RL. The rodent estrous cycle: characterization of vaginal cytology and its utility in toxicological studies. Birth Defects Res B Dev Reprod Toxicol. 2007;80:84-97.

28. Li S, Davis B. Evaluating rodent vaginal and uterine histology in toxicity studies. Birth Defects Res B Dev Reprod Toxicol. 2007;80:246-52.

29. Kurita T. Developmental origin of vaginal epithelium. Differentiation. 2010;80:99-105.

30. Nam EJ, Kim JW, Hong JW, Jang HS, Lee SY, Jang SY, et al. Expression of the p16 and Ki-67 in relation to the grade of cervical intraepithelial neoplasia and high-risk human papillomavirus infection. J Gynecol Oncol. 2008;19:162-8.

31. Tsoumpou I, Arbyn M, Kyrgiou M, Wentzensen N, Koliopoulos G, Martin-Hirsch P, et al. p16 (INK4a) immunostaining in cytological and histological specimens from the uterine cervix: a systematic review and meta-analysis. Cancer Treat Rev. 2009;35:210-20.

32. Zhong P, Li J, Gu Y, Liu Y, Wang A, Sun Y, et al. P16 and Ki-67 expression improves the diagnostic accuracy of cervical lesions 
but not predict persistent high risk human papillomavirus infection with CIN1. Int J Clin Exp Pathol. 2015;8:2979-86.

33. Li Y, Liu J, Gong L, Sun X, Long W, Combining HPV. DNA load with p16/Ki-67 staining to detect cervical precancerous lesions and predict the progression of CIN1-2 lesions. Virol J. 2019;16:117.

34. Butt AJ, McNeil CM, Musgrove EA, Sutherland RL. Downstream targets of growth factor and oestrogen signalling and endocrine resistance: the potential roles of c-Myc, cyclin D1 and cyclin E. Endocr Relat Cancer. 2005;12:S47-59.

35. Li XJ, Guo QH, Wang X, Xue B, Sun LQ, Meng QT, et al. LRP16 gene protects mouse insulinoma MIN6 cells against fatty acidinduced apoptosis through Akt/FoxO1 signaling. Chin Med J. 2012;125:1695-702.

36. Scheffner M, Huibregtse JM, Vierstra RD, Howley PM. The HPV-16 E6 and E6-AP complex functions as a ubiquitin-protein ligase in the ubiquitination of p53. Cell. 1993;75:495-505.

37. Chellappan S, Kraus VB, Kroger B, Howley PM, Phelps WC, Nevins JR. Adenovirus E1A, simian virus 40 tumor antigen, and human papillomavirus E7 protein share the capacity to disrupt the interaction between transcription factor $\mathrm{E} 2 \mathrm{~F}$ and the retinoblastoma gene product. Proc Natl Acad Sci USA. 1992;89:4549-53.

38. Duensing S, Münger K. The human papillomavirus type 16 E6 and E7 oncoproteins independently induce numerical and structural chromosome instability. Cancer Res. 2002;62:7075-82.

39. Duensing S, Münger K. Mechanisms of genomic instability in human cancer: insights from studies with human papillomavirus oncoproteins. Int J Cancer. 2004;109:157-62.

40. Riley RR, Duensing S, Brake T, Münger K, Lambert PF, Arbeit JM. Dissection of human papillomavirus E6 and E7 function in transgenic mouse models of cervical carcinogenesis. Cancer Res. 2003;63:4862-71.

41. Shai A, Brake T, Somoza C, Lambert PF. The human papillomavirus E6 oncogene dysregulates the cell cycle and contributes to cervical carcinogenesis through two independent activities. Cancer Res. 2007;67:1626-35.

42. Chen M, Li L, Zheng PS. SALL4 promotes the tumorigenicity of cervical cancer cells through activation of the Wnt $/ \beta$-catenin pathway via CTNNB1. Cancer Sci. 2019;110:2794-805.
43. Suzuki E, Chiba T, Yokosuka O. Oncofetal gene SALL4 in aggressive hepatocellular carcinoma. $N$ Engl J Med. 2013;369:1170-1.

44. Yong KJ, Li A, Ou WB, Hong CKY, Zhao W, Wang F, et al. Targeting SALL4 by entinostat in lung cancer. Oncotarget. 2016;7:75425-40.

45. Yuan X, Zhang X, Zhang W, Liang W, Zhang P, Shi H, et al. SALL4 promotes gastric cancer progression through activating CD44 expression. Oncogenesis. 2016;5:e268.

46. Kobayashi D, Kuribayshi K, Tanaka M, Watanabe N. SALL4 is essential for cancer cell proliferation and is overexpressed at early clinical stages in breast cancer. Int J Oncol. 2011;38:933-9.

47. Li A, Jiao Y, Yong KJ, Wang F, Gao C, Yan B, et al. SALL4 is a new target in endometrial cancer. Oncogene. 2015;34:63-72.

48. Gu TT, Liu SY, Zheng PS. Cytoplasmic NANOG-positive stromal cells promote human cervical cancer progression. Am J Pathol. 2012;181:652-61.

49. Wang YD, Cai N, Wu XL, Cao HZ, Xie LL, Zheng PS. OCT4 promotes tumorigenesis and inhibits apoptosis of cervical cancer cells by miR-125b/BAK1 pathway. Cell Death Dis. 2013;4:e760.

50. Chen Q, Cao HZ, Zheng PS. LGR5 promotes the proliferation and tumor formation of cervical cancer cells through the Wnt/ $\beta$-catenin signaling pathway. Oncotarget. 2014;5:9092-105.

51. Chen Q, Zheng PS, Yang WT. EZH2-mediated repression of GSK-3 $\beta$ and TP53 promotes Wnt $/ \beta$-catenin signaling-dependent cell expansion in cervical carcinoma. Oncotarget. 2016;7:36115-29.

52. Hong K, Choi Y. Role of estrogen and RAS signaling in repeated implantation failure. BMB Rep. 2018;51:225-9.

53. Miyagawa S, Iguchi T. Epithelial estrogen receptor 1 intrinsically mediates squamous differentiation in the mouse vagina. Proc Natl Acad Sci USA. 2015;112:12986-91.

54. Brake T, Lambert PF. Estrogen contributes to the onset, persistence, and malignant progression of cervical cancer in a human papillomavirus-transgenic mouse model. Proc Natl Acad Sci USA. 2005;102:2490-5.

55. Zang L, Hong Q, Yang G, Gu W, Wang A, Dou J, et al. MACROD1/LRP16 enhances LPS-stimulated inflammatory responses by up-regulating a Rac1-dependent pathway in adipocytes. Cell Physiol Biochem. 2018;51:2591-603. 